\title{
Neoadjuvant chemoradiotherapy followed by surgery for esophageal adenocarcinoma: Significance of microscopically positive circumferential radial margins
}

\author{
John A. Harvin, MD, Guy Lahat, MD, Arlene M. Correa, PhD, Jared Lee, MS, Dipen Maru, MD, \\ Jaffer Ajani, MD, Edith M. Marom, MD, James Welsh, MD, Manoop S. Bhutani, MD, Garret Walsh, MD, \\ Jack Roth, MD, Reza Mehran, MD, Ara Vaporciyan, MD, David Rice, MD, Stephen Swisher, MD, and \\ Wayne Hofstetter, MD
}

\begin{abstract}
Objectives: The incidence and consequence of an isolated involved circumferential radial margin (CRM) after resection for esophageal adenocarcinoma in the setting of neoadjuvant chemoradiotherapy (CRT) has not been reported. We aimed to determine the frequency and significance of a close $(<1 \mathrm{~mm})$ or involved CRM in patients undergoing esophagectomy after CRT.
\end{abstract}

\begin{abstract}
Methods: We retrospectively analyzed the data from patients undergoing resection from 1997 to 2008 for esophageal adenocarcinoma after neoadjuvant CRT. A positive CRM was defined as microscopic tumor at or less than $1 \mathrm{~mm}$ of the radial margin. An R1 resection was tumor at the radial margin. Only patients with ypT3 or greater tumors were included. R2 resections were excluded. Statistical comparisons were performed using Cox regression and Kaplan-Meier analyses.
\end{abstract}

Results: A total of 160 patients met the inclusion criteria, $42(26 \%)$ had a positive CRM. The median survival did not significantly differ between the CRM-negative and -positive groups ( 28 vs 50 months, $P=.84$ ). A propensity score matching analysis also failed to find a significant difference in outcomes. When analyzed by tumor present at the margin (R1), R0 patients had a longer median survival compared with R1 patients ( 28 vs 8 months, $P=.01)$. This difference, however, was not seen on propensity score matching.

Conclusions: Resections of locally advanced esophageal adenocarcinoma with residual transmural viable tumor after CRT frequently showed involvement of the radial margin with tumor either close to or at the margin. Tumor close $(<1 \mathrm{~mm})$ to the radial margin did not result in a significant decrease in overall or disease-free survival or increase in local recurrence. (J Thorac Cardiovasc Surg 2012;143:412-20)

The significance of circumferential radial margin (CRM) status in esophageal cancer first gained attention after it was found that a positive CRM in rectal cancer purported greater local recurrence rates. Controversy exists regarding the significance of a close $(<1 \mathrm{~mm})$ or microscopically positive CRM after esophagectomy for esophageal cancer. Most studies have shown that a close or involved CRM is associated with shorter overall survival in patients who have undergone surgery alone or neoadjuvant chemotherapy. ${ }^{1-5}$ However, studies of the incidence and significance of a positive CRM in patients with adenocarcinoma

From the Department of Thoracic and Cardiovascular Surgery, University of Texas M.D. Anderson Cancer Center, Houston, Tex.

Disclosures: Authors have nothing to disclose with regard to commercial support.

Read at the 91st Annual Meeting of The American Association for Thoracic Surgery, Philadelphia, Pennsylvania, May 7-11, 2011.

Received for publication April 21, 2011; revisions received Sept 6, 2011; accepted for publication Oct 20, 2011; available ahead of print Dec 15, 2011.

Address for reprints: Wayne Hofstetter, MD, Department of Thoracic and Cardiovascular Surgery, University of Texas M.D. Anderson Cancer Center, 1515 Holcombe Boulevard, No.445, Houston, TX 77030 (E-mail: Wayne.Hofstetter@mdanderson. org).

0022-5223/\$36.00

Copyright (c) 2012 by The American Association for Thoracic Surgery

doi:10.1016/j.jtcvs.2011.10.044 treated with neoadjuvant chemotherapy and concurrent radiotherapy (CRT) are lacking.

Currently, there are two definitions of a positive esophageal CRM. First, the College of American Pathologists (CAP) has defined a positive CRM as tumor at the cut margin of resection. Alternatively, the Royal College of Pathologists (RCP) consider tumor at or within $1 \mathrm{~mm}$ of the margin to be positive. Of particular relevance, most published, peer-reviewed studies aiming to define the effect of a positive CRM have used the RCP definition.

CRT before esophagectomy has been shown to improve local control and survival compared with surgery alone. ${ }^{6-8}$ CRT has been shown to induce a complete oncologic response in approximately $25 \%$ to $35 \%$ of patients with esophageal cancer and to significantly improve the R0 resection rate. ${ }^{9,10}$ Currently, trimodality therapy (ie, neoadjuvant CRT followed by surgery) has been increasingly used in the treatment of esophageal cancer.

The purpose of the present study was to determine the frequency and outcome of a positive CRM in patients undergoing esophagectomy after CRT in an off-protocol setting. To analyze patients with a similar risk of a positive CRM, we focused our study on patients who were found 


\author{
Abbreviations and Acronyms \\ $\mathrm{CAP}=$ College of American Pathologists \\ $\mathrm{CI}=$ confidence interval \\ $\mathrm{CRM}=$ circumferential radial margin \\ $\mathrm{CRT}=$ concurrent radiotherapy \\ HR = hazard ratio \\ $\mathrm{RCP}=$ Royal College of Pathologists
}

to have post-treatment pathologic transmural viable tumor (ypT3 or greater).

\section{MATERIALS AND METHODS}

Patients were selected from a comprehensive, prospective database that includes all patients undergoing surgical resection for esophageal cancer at the University of Texas M.D. Anderson Cancer Center. Data are entered during the preoperative evaluation, at the time of operation, on the day of discharge, and at every postoperative outpatient encounter. The present study was a retrospective review of that database and has been approved by the University of Texas M.D. Anderson Cancer Center institutional review board; the requirement of individual consent was waived.

All patients who underwent esophagectomy at the University of Texas M.D. Anderson Cancer Center from January 1, 1997 to December 31, 2008 and who received neoadjuvant chemoradiotherapy for middle to distal esophageal adenocarcinoma and gastroesophageal junction type I and II were included. The patients who underwent emergent operations, reoperations, or $\mathrm{R} 2$ resections or who died during the perioperative hospitalization were excluded. Patients with unknown nodal status or systemic metastases were also excluded. Thus, our cohort included only patients with ypT3 or greater tumors.

Because we sought to evaluate the effect of an isolated, positive CRM, the patients with positive proximal and distal margins were also excluded. Therefore, the CRM-negative group contained patients with no positive margins and the CRM-positive group has isolated, positive radial margins.

For the purposes of the present study, a positive CRM was defined in agreement with the RCP definition (ie, tumor at or within $1 \mathrm{~mm}$ of the radial margin). However, for clarity of data collection and differentiation, we defined an R1 resection as tumor at the radial margin, similar to the CAP criteria.

The demographic, patient, and tumor characteristics were recorded. These included age; body mass index; gender; race; number of positive lymph nodes; American Joint Committee on Cancer, 6th edition, clinical stage; American Joint Committee on Cancer, 7th edition, pathologic stage; American Society of Anesthesiologists risk score; peripheral vascular disease; coronary artery disease; diabetes; renal insufficiency; alcohol abuse ( $\geq 4 \mathrm{oz} / \mathrm{d}$ or equivalent); tobacco use; congestive heart failure; chronic obstructive pulmonary disease (requiring chronic bronchodilator use); weight loss; grade (differentiation) of adenocarcinoma; and proximal, distal, and radial margin status.

These variables were summarized with the use of descriptive characteristics and compared with the use of $t$ tests and chi-square analyses. Cox regression analysis with stepwise backward elimination was used for univariate and multivariate analyses. First, univariate analysis of all characteristics was performed. The variables with $P<.25$ on univariate analysis were then entered into a multivariate logistic regression model. Significance in the multivariate analysis was defined as $P<.05$. Propensity matched analyses were performed for both ypT3 CRM-positive and R1 subgroups.
The primary outcome was survival, defined as the interval from surgery to death. The secondary outcomes include local recurrence and disease-free survival. These outcomes were analyzed using Kaplan-Meier analysis and the log-rank test.

\section{RESULTS \\ Incidence in the Cohort}

A total of 160 patients during the study period met our previously stated inclusion criteria and had a pathologic classification of ypT3 or greater (ypT3+). Of these $160 \mathrm{pa}-$ tients, $42(26 \%)$ had an isolated, positive CRM. Also, 8 patients with ypT3+ $(5 \%)$ underwent $\mathrm{R} 1$ resection solely because of tumor at the radial margin (ie, proximal and distal margins were negative). The demographic, patient, and tumor characteristics of the CRM-negative and -positive groups were comparable and are listed in Table 1.

\section{Survival by RCP Criteria}

A positive CRM was not significant on univariate analysis for survival (hazard ratio [HR], 0.95; 95\% confidence interval $[\mathrm{CI}], 0.56-1.60)$. On multivariate analysis, the number of positive lymph nodes (HR, 1.10; 95\% CI, 1.04-1.17) and clinical stage IV (HR, 2.86; 95\% CI, 1.30-6.26) emerged as independent predictors of survival (Table 2).

The median and 5-year survival for the ypT3+ CRM-negative (28 months and $25 \%$, respectively) and CRM-positive (50 months and $43 \%$, respectively) also did not differ significantly $(P=.84$; Figure 1$)$.

\section{Local Recurrence Using RCP Criteria}

Of the 160 patients, 11 (7\%) developed local recurrence, defined as tumor occurring within the esophageal or gastric remnant. Of the 11 local recurrences, 7 (4\%; range, 5-39 months) were CRM negative and 4 (2\%; range, 6-32 months) were CRM positive. The incidence of local recurrence was not significantly different between the two groups $(P=.16$; Figure 1$)$.

A positive CRM was not significant on univariate analysis for local recurrence (HR, 2.36; 95\% CI, 0.69-8.11). Multivariate analysis revealed that the number of positive lymph nodes (HR, 1.43; 95\% CI, 1.19-1.72), tumor location (HR, 0.01; 95\% CI, 0.00-0.6), and alcohol abuse (HR, 5.15; 95\% CI, 1.36-19.47) were independent predictors of local recurrence (Table 2).

\section{Disease-Free Survival Using RCP Criteria}

A CRM was also not significant (HR, 0.78; 95\% CI, $0.48-1.27$ ) on univariate analysis for disease-free survival. Multivariate analysis revealed that the ypN descriptor (ypN2, HR, 1.82; 95\% CI, 1.05-3.13; ypN3, HR, 3.46; $95 \%$ CI, 1.71-6.98) was an independent predictor of disease-free survival (Table 2). 
TABLE 1. Demographic, patient and tumor characteristics of cohort by CRM status using RCP criteria

\begin{tabular}{|c|c|c|c|}
\hline Variable & $\begin{array}{c}\text { Negative } \\
\text { CRM }\end{array}$ & $\begin{array}{c}\text { Positive } \\
\text { CRM }\end{array}$ & $\begin{array}{c}P \\
\text { value }\end{array}$ \\
\hline Patients (n) & 118 & 42 & \\
\hline $\operatorname{Age}^{*}(\mathrm{y})$ & & & .98 \\
\hline Mean & 60 & 61 & \\
\hline Range & $23-80$ & $37-84$ & \\
\hline Positive lymph nodes (n) & & & 11 \\
\hline Median*,, & 0 & 1 & \\
\hline Range & $0-20$ & $0-14$ & \\
\hline Gender & & & 1.00 \\
\hline Male & $108(92)$ & $39(92)$ & \\
\hline Female & $10(8)$ & $3(8)$ & \\
\hline Race & & & .20 \\
\hline White & $110(93)$ & $36(86)$ & \\
\hline Other & $8(7)$ & $6(14)$ & \\
\hline ASA score & & & 1.00 \\
\hline $1-2$ & $27(23)$ & $10(24)$ & \\
\hline $3-4$ & $91(77)$ & $32(76)$ & \\
\hline Preoperative radiation dose (cGy) & & & .02 \\
\hline$\leq 45$ & $47(40)$ & $8(19)$ & \\
\hline$>45$ & $71(60)$ & $34(81)$ & \\
\hline Surgery type & & & .25 \\
\hline Ivor Lewis & $84(71)$ & $25(60)$ & \\
\hline Transhiatal & $16(14)$ & $6(14)$ & \\
\hline Three-field & $12(10)$ & $5(12)$ & \\
\hline Minimally invasive & $6(5)$ & $6(14)$ & \\
\hline Resection extent*,$\ddagger$ & & & .00 \\
\hline R0 & $118(100)$ & $34(81)$ & \\
\hline R1 & $0(0)$ & $8(19)$ & \\
\hline Pathologic N status $\ddagger$ & & & .37 \\
\hline No & $62(52)$ & $16(38)$ & \\
\hline N1 & $32(27)$ & $15(36)$ & \\
\hline $\mathrm{N} 2$ & $15(13)$ & $8(19)$ & \\
\hline N3 & $9(8)$ & $3(7)$ & \\
\hline Pathologic stage (AJCC 7th edition) & & & .37 \\
\hline IIB & $62(53)$ & $16(38)$ & \\
\hline IIIA & $32(27)$ & $15(36)$ & \\
\hline IIIB & $15(13)$ & $8(19)$ & \\
\hline IIIC & $9(8)$ & $3(7)$ & \\
\hline Grade (differentiation) & & & .84 \\
\hline Well/moderately & $32(28)$ & $10(25)$ & \\
\hline Poorly/undifferentiated & $82(72)$ & $30(75)$ & \\
\hline Tumor location $\dagger$ & & & 1.00 \\
\hline Middle & $4(3)$ & $1(2)$ & \\
\hline Lower/GEJ & $114(97)$ & $41(98)$ & \\
\hline
\end{tabular}

Data presented as numbers, with percentages in parentheses. $C R M$, Circumferential radial margin; ASA, American Society of Anesthesiologists; AJCC, American Joint Committee on Cancer; GEJ, gastroesophageal junction. *Carried into multivariate analysis for survival outcome; also included in multivariate analysis, but not above: clinical stage. †Carried into multivariate analysis for local recurrence outcome; also included in multivariate analysis, but not above: alcohol abuse. $\ddagger$ Carried into multivariate analysis for disease-free survival outcome.

The median and 5-year disease-free survival for the CRM-negative group (16 months and $25 \%$, respectively) and CRM-positive group (18 months and $41 \%$, respectively) did not differ significantly $(P=.31$; Figure 1$)$.

\section{Propensity Score Match Using RCP Criteria}

A propensity score match for the patients with ypT3+ was also performed. Forty-four pairs of the ypT3+ subgroup were matched by age, number of lymph nodes checked, number of positive lymph nodes, body mass index, gender, race, tumor location, type of esophageal surgery, clinical stage, American Society of Anesthesiologists score, peripheral vascular disease, coronary artery disease, alcohol abuse, congestive heart failure, chronic obstructive pulmonary disease, recent weight loss, diabetes, tobacco use, and pathologic N status. No statistically significant difference was found in survival $(P=.97)$, local recurrence $(P=.23)$, or disease-free survival $(P=.52)$ between patients with or without an involved CRM using the RCP criteria.

\section{Survival Using CAP Criteria}

The median and 5-year survival for the R0 patients was significantly longer than the R1 patients (28 versus 8 months and $28 \%$ vs $19 \%$, respectively; $P=.01$; Figure 2 ).

\section{Local Recurrence Using CAP Criteria}

Of the 11 local recurrences, $10(6 \%)$ were in the R0 group and $1(1 \%)$ in the R1 group. No statistically significant difference was found in local recurrence between the two groups $(P=.17$; Figure 2$)$.

\section{Disease-Free Survival Using CAP Criteria}

The median and 5-year disease-free survival for the R0 group was also significantly longer than that of the R1 group (18 vs 8 months and $29 \%$ vs $0 \%$, respectively; $P=.02$; Figure 2).

\section{Propensity Score Match Using CAP Criteria}

Again, we performed a propensity score match, but this time compared the outcomes using the CAP criteria for a positive radial margin. Eight pairs were matched by age, number of lymph nodes checked, number of positive lymph nodes, body mass index, gender, race, tumor location, type of esophageal surgery, clinical stage, American Society of Anesthesiologists score, peripheral vascular disease, coronary artery disease, alcohol abuse, congestive heart failure, recent weight loss, diabetes, tobacco use, radiation dose, and pathologic $\mathrm{N}$ status. No statistically significant difference was found in survival $(P=.97)$, local recurrence $(P=.38)$, or disease-free survival $(P=.14)$ between patients with an involved CRM using the CAP criteria (Figure 2).

\section{Multivariate Analysis for Outcomes: Involved CRM and R1 Resection}

Finally, we performed univariate and multivariate analyses for independent predictors of close (CRM positive) and 
TABLE 2. Results of univariate and multivariate analysis of cohort for outcomes: overall and disease-free survival and local recurrence

\begin{tabular}{|c|c|c|c|c|c|}
\hline \multicolumn{6}{|c|}{ Factors significant on univariate analysis } \\
\hline \multicolumn{2}{|c|}{ Overall survival } & \multicolumn{2}{|c|}{ Disease-free survival } & \multicolumn{2}{|c|}{ Local recurrence } \\
\hline Variable & HR $(95 \%$ CI $)$ & Variable & HR $(95 \%$ CI $)$ & Variable & HR $(95 \%$ CI $)$ \\
\hline Age & $1.02(1.00-1.04)$ & Age & $1.01(1.00-1.03)$ & $\mathrm{CHF}$ & $36.09(3.19-407.87)$ \\
\hline Positive lymph nodes & $1.11(1.05-1.18)$ & Positive lymph nodes & $1.10(1.05-1.16)$ & Positive lymph nodes & $1.29(1.11-1.49)$ \\
\hline BMI $\geq 25 \mathrm{~kg} / \mathrm{m}^{2}$ & $0.77(0.51-1.19)$ & ASA score $3-4$ & $1.62(0.96-2.73)$ & $\mathrm{BMI} \geq 25 \mathrm{~kg} / \mathrm{m}^{2}$ & $0.27(0.08-0.92)$ \\
\hline Female gender & $0.60(0.26-1.38)$ & Clinical stage III & $1.45(0.92-2.28)$ & White race & $0.29(0.08-1.08)$ \\
\hline & & Clinical stage IV & $2.00(0.96-4.19)$ & & \\
\hline Lower tumor location & $0.43(0.16-1.18)$ & ypN2 & $1.81(1.05-3.11)$ & Lower tumor location & $0.02(0.00-0.11)$ \\
\hline & & ypN3 & $3.56(1.95-7.64)$ & & \\
\hline Clinical stage III & $1.45(0.89-2.36)$ & Weight loss in past $3 \mathrm{mo}$ & $1.27(0.86-1.89)$ & Alcohol use $\geq 4 \mathrm{oz} / \mathrm{d}$ & $3.88(1.18-12.75)$ \\
\hline Clinical stage IV & $2.36(1.10-5.11)$ & & & & \\
\hline ASA 3-4 & $1.85(1.03-3.33)$ & Diabetes & $1.35(0.83-2.21)$ & CRM Positive & $2.36(0.69-8.11)$ \\
\hline $\mathrm{CHF}$ & $2.71(0.85-8.67)$ & $\mathrm{R} 1$ resection & $2.48(1.14-5.39)$ & $\mathrm{R} 1$ resection & $3.84(0.48-30.98)$ \\
\hline Weight loss in past $3 \mathrm{mo}$ & $1.33(0.88-2.02)$ & & & ypN2 & $5.69(1.23-26.40)$ \\
\hline & & & & ypN3 & $11.95(1.86-76.86)$ \\
\hline $\mathrm{R} 1$ resection & $2.91(1.26-6.74)$ & & & & \\
\hline ypN2 & $2.03(1.14-2.57)$ & & & & \\
\hline ypN3 & $3.19(1.57-6.47)$ & & & & \\
\hline
\end{tabular}

\begin{tabular}{lccccc}
\hline \multicolumn{7}{c}{ Factors significant on multivariate analysis } \\
\hline Positive lymph nodes & $1.10(1.04-1.17), P=.00$ & $\mathrm{ypN} 2$ & $1.82(1.05-3.13), P=.03$ & Positive lymph nodes & $1.43(1.19-1.72), P=.00$ \\
& & $\mathrm{ypN3}$ & $3.46(1.71-6.98), P=.00$ & & \\
& & & & Lower tumor location & $0.01(0.00-0.06), P=.00$ \\
Clinical stage IV & $2.86(1.30-6.26), P<.01$ & & & Alcohol use $\geq 4 \mathrm{oz} / \mathrm{d}$ & $5.15(1.36-19.47), P=.02$ \\
& & & &
\end{tabular}

Variables included in univariate analysis are listed in the "Materials and Methods" section. Variables with $P<.25$ on univariate analysis were included multivariate analysis. $H R$, Hazard ratio; $C I$, confidence interval; $C H F$, congestive heart failure; BMI, body mass index; ASA, American Society of Anesthesiologists.

involved radial margins (R1). The patient variables included in the univariate and multivariate analyses for survival, local recurrence, and disease-free survival were included in this analysis.

The patient variables found to be significantly predictive of a positive CRM included a history of congestive heart failure (odds ratio [OR], 12.39; 95\% CI, 1.19-128.95) and a history of tobacco use (OR, 0.44; 95\% CI, 0.21-0.95).

The independent predictors of an $\mathrm{R} 1$ resection included the numbers of lymph nodes checked (OR, $0.87 ; 95 \% \mathrm{CI}$, $0.77-0.98$ ); presence of peripheral vascular disease (OR, 26.17; 95\% CI, 1.26-544.20); and American Joint Committee on Cancer, 7th edition, pathologic stage III (OR, 26.90; $95 \%$ CI, 1.59-454.89).

\section{DISCUSSION}

In summary, no difference in survival, local recurrence, or disease-free survival was seen when defining a radial margin using the RCP criteria. When using the CAP definition for a positive radial margin, the cohort appeared to have a significantly lower overall and disease-free survival. This difference, however, was not seen on a propensity score match analysis.

Our results have shown that in a group of patients with adenocarcinoma of the esophagus who undergo resection after neoadjuvant chemoradiotherapy, the incidence
$(26 \%)$ of a surgically positive CRM as defined by the $\mathrm{RCP}$ is a frequent event. In this same group of patients, a positive radial margin using the CAP definition is an infrequent event $(5 \%)$.

There are several potential reasons for this to occur. The addition of neoadjuvant chemoradiotherapy is known to improve the chances of an $\mathrm{R} 0$ resection. ${ }^{11}$ We believe that for patients with locally advanced disease, the addition of neoadjuvant multimodality therapy facilitates the course of the operation, allowing the surgeon to more easily attain a complete resection after a size reduction of the tumor brought about by the additional local therapy. However, not all patients respond equally to therapy. Although all the patients in the ypT3+ subgroup had residual viable tumor, those with tumor at the margin might represent patients with more advanced disease or whose tumors were more resistant to neoadjuvant treatment.

Theoretically, the type of surgical resection can affect the radial margin outcome, although in our study population, we found no correlation between the resection type and a positive radial margin. It is our policy to widely resect at the area at which the tumor is at risk of radial invasion. This includes resection of structures such as the pleura, diaphragm, pericardium, periaortic fat, and so forth. Examination of our data would note the infrequent use of procedures that do not involve intrathoracic dissection. 

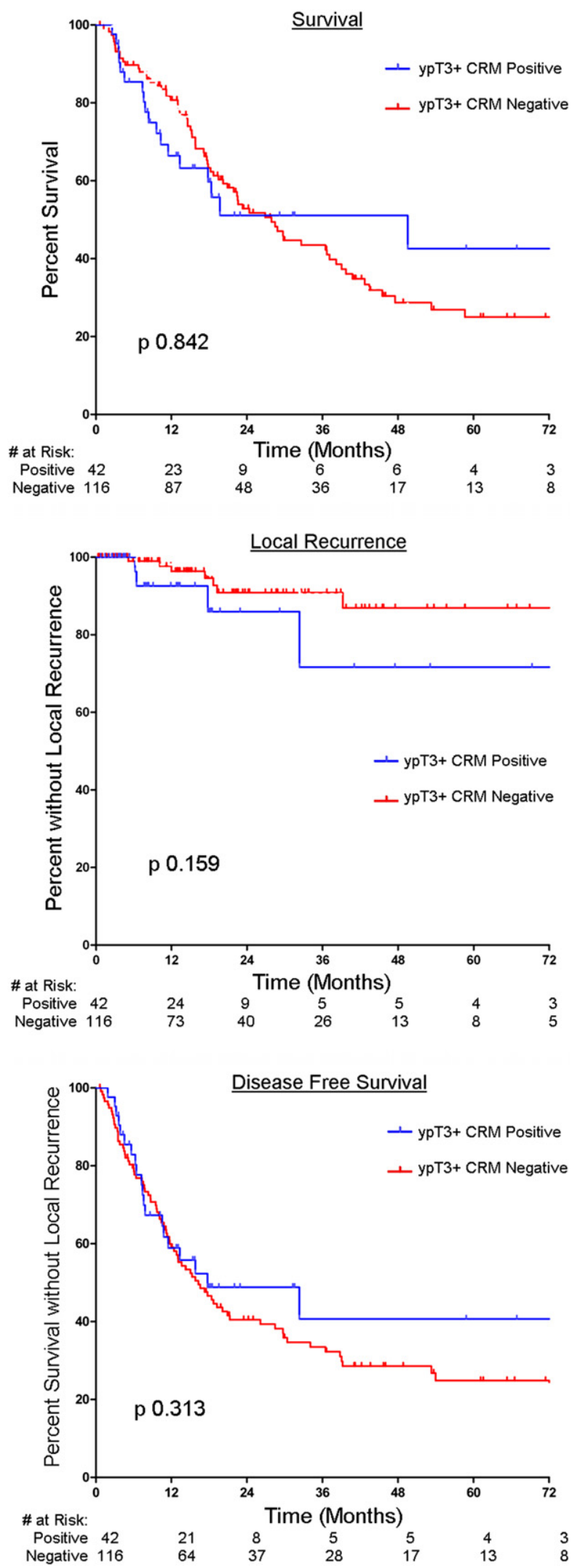

FIGURE 1. Kaplan-Meier curves for outcomes of cohort according to Royal College of Pathologists $(R C P)$ criteria.
Given that locoregional recurrence can be a significant risk even after chemoradiotherapy and surgery, we have worried about the utility of less-aggressive resection. ${ }^{8}$ Finally, fullthickness lesions of the esophagus can involve structures surrounded by a potential space, such as the pleura or peritoneal cavity. In these cases, the surgeon has no control over the final radial margin evaluation.

Although the occurrence of a positive CRM using the $\mathrm{RCP}$ criteria in our study was fairly frequent, the rate compares favorably with that from other studies by Dexter and colleagues, ${ }^{3}$ Sagar and colleagues, ${ }^{4}$ Scheepers and colleagues, ${ }^{2}$ Saha and colleagues, ${ }^{1}$ and Sujendran and colleagues. ${ }^{5}$ In these studies, the patients either received no neoadjuvant therapy or neoadjuvant chemotherapy, or, in the case of one study, neoadjuvant chemoradiotherapy was given to a small minority of the total study population. Those investigators described positive CRM rates of $36 \%$ to $55 \%$, and all found that a positive CRM, using the RCP criteria, to be an independent predictor of survival.

Our results are important for several reasons. First, these results contradict the findings of multiple studies that reviewed a close or involved CRM (RCP criteria). Dexter and colleagues, ${ }^{3}$ Sagar and colleagues, ${ }^{4}$ Scheepers and colleagues, ${ }^{2}$ Saha and colleagues, ${ }^{1}$ and Sujendran and colleagues $^{5}$ all found a close or positive CRM to be independent predictors of survival and/or local recurrence. Only Khan and colleagues ${ }^{12}$ failed to find this relationship. Our results also contradict a recent study that indicated CRM using the RCP criteria is a significant factor associated with survival in patients who received neoadjuvant chemoradiotherapy for squamous cell carcinoma of the esophagus. ${ }^{13}$

Second, the more clinically relevant definition of a positive CRM might be tumor at the radial margin rather than very close to it. Our data indicated that tumor close to the margin is not a significant factor for survival, local recurrence, or disease-free survival. These results echo the findings from Deeter and colleagues ${ }^{14}$ indicating that the CAP definition of a positive CRM was more clinically meaningful than the RCP definition. We believe that the lack of a significant difference between outcomes in the propensity score match analysis of the R1 and R0 groups resulted from underpowering. Additional studies with a larger number of ypT3+R1 patients are needed to delineate the importance of tumor at the radial margin in patients undergoing esophagectomy after CRT.

Finally, these data provide some clinically relevant information. After surgery in which the surgeon believes a complete resection has been have performed, the report of a positive CRM as defined by the RCP criteria can easily confuse both the patient and the clinician. According to our results, the report of tumor close (within $1 \mathrm{~mm}$ ) of the radial margin in patients who received CRT does not portend decreased overall and disease-free survival or a greater incidence of local recurrence. 

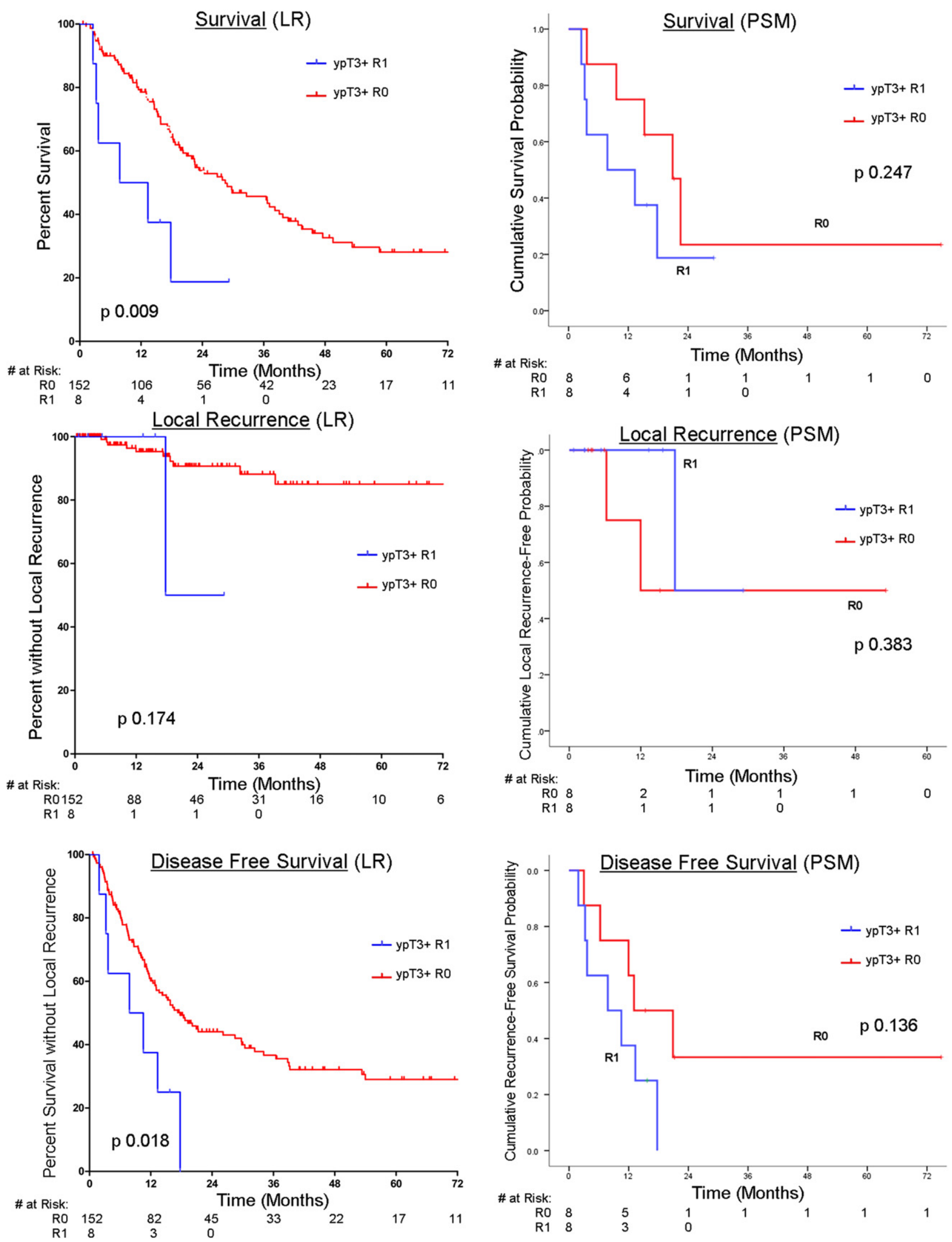

FIGURE 2. Kaplan-Meier curves for outcomes of cohort according to College of American Pathologists (CAP) criteria by log-rank test and propensity score match. LR, Log rank; PSM, propensity score matching. 
However, the present study had some limitations. First, the CRM-positive and R1 radial margin groups (44 and 8 patients, respectively) were relatively small. This could have led to underpowering, especially in the $\mathrm{R} 1$ group analyses in which a type II error might have been present. Two, the pathologic evaluation of the specimens is often performed by a single pathologist without secondary verification. Third, just as with any retrospective study, selection bias is a concern. The propensity score match was performed in an attempt to mitigate this sort of bias. Fourth, it is difficult to establish a specific protocol for pathologic fixation of specimens that simultaneously involve several body cavities. Thus, there could be times when the surgeon performs what they perceive to have been an R0 resection, but a positive CRM is found because the envelope of surrounding pleura or soft tissue was disrupted.

\section{CONCLUSIONS}

A close $(<1 \mathrm{~mm})$ radial margin in patients undergoing esophagectomy after neoadjuvant chemoradiotherapy does not appear to be an independent predictor of survival, local recurrence, or disease-free survival. An involved radial margin, however, might be the more clinically relevant definition. Future studies concerning multimodality therapy with chemoradiotherapy and surgery should include the CAP definition of radial margin involvement.

\section{References}

1. Saha AK, Sutton C, Olorunda R, Dexter S, Sue-Ling H, Sarela AI. Neoadjuvant chemotherapy and surgery for esophageal adenocarcinoma: Prognostic value of circumferential resection margin and stratification of N1 category. Ann Surg Oncol. 2009; 16:1364-70.

2. Scheepers JJG, van der Peet DL, Veenhof AAFA, Cuesta MA. Influence of circumferential resection margin on prognosis in distal esophageal and gastroesophageal cancer approached through the transhiatal route. Dis Esophagus. 2009;22:42-8.

3. Dexter SPL, Sue-Ling H, McMahon MJ, Mapstone N, Margin IG. Circumferential resection margin involvement: An independent predictor of survival following surgery for oesophageal cancer. Gut. 2001;48:667-70.

4. Sagar PM, Johnston D, McMahon MJ, Dixon MF, Quirke P. Significance of circumferential resection margin involvement after oesophagectomy for cancer. $\mathrm{Br}$ J Surg. 1993;80:1386-8.

5. Sujendran V, Wheeler J, Baron R, Warren BF, Maynard N. Effect of neoadjuvant chemotherapy on circumferential margin positivity and its impact on prognosis in patients with resectable oesophageal cancer. Br J Surg. 2008;95:191-4.

6. Walsh TN, Noonan N, Hollywood D, Kelly A, Keeling N, Hennessy TPJ. A comparison of multimodal therapy and surgery for esophageal adenocarcinoma. N Engl J Med. 1996;335:462-7.

7. Tepper JE, Krasna MJ, Niedzwiecki D, Hollis D, Reed CE, Goldberg R, et al. Phase III trial of trimodality therapy with cisplatin, fluorouracil, radiotherapy, and surgery compared with surgery alone for esophageal cancer: CALGB 9781. J Clin Oncol. 2008;26:1086-92.

8. Urba SG, Orringer MB, Turrisi A, Iannettoni M, Forastiere A, Strawderman M. Randomized trial of preoperative chemoradiation versus surgery alone in patients with locoregional esophageal carcinoma. J Clin Oncol. 2001;19:305-13.

9. Adelstein DJ, Rice TW, Becker M, Larto MA, Kirby TJ, Koka A, et al. Use of concurrent chemotherapy, accelerated fractionation radiation, and surgery for patients with esophageal carcinoma. Cancer. 1997;80:1011-20.

10. Hofstetter W, Swisher SG, Correa AM, Hess K, Putnam JB, Ajani JA, et al. Treatment outcomes of resected esophageal cancer. Ann Surg. 2002;136:376-84.

11. Burmeister BH, Smithers BM, Gebski V, Fitzgerald L, Simes RJ, Devitt P, et al. Surgery alone versus chemoradiotherapy followed by surgery for resectable can- cer of the oesophagus: A randomized controlled phase III trial. Lancet Oncol. 2005;6:659-68.

12. Khan OA, Fitzgerald JJ, Soomro I, Beggs FD, Morgan WE, Duffy JP. Prognostic significance of circumferential resection margin involvement following oesophagectomy for cancer. Br J Cancer. 2003;88:1549-52.

13. Chao YK, Yeh CJ, Chang HK, Tseng CK, Chu YY, Hsieh MJ, et al. Impact of circumferential resection margin distance on locoregional recurrence and survival after chemoradiotherapy in esophageal squamous cell carcinoma. Ann Surg Oncol. 2011;18:529-34.

14. Deeter M, Dorer R, Kuppusamy MK, Koehler RP, Low DE. Assessment of criteria and clinical significance of circumferential resection margins in esophageal cancer. Arch Surg. 2009;144:618-24.

\section{Discussion}

Dr Gail E. Darling (Toronto, Ontario, Canada). I want to thank Dr Harvin for an excellent presentation and for providing me with the manuscript well in advance of the meeting.

You have addressed a very important question regarding positive circumferential radial margins, and, specifically, you have addressed the problem of two conflicting definitions. Your results have shown that the Royal College of Pathologists' definition has no predictive value in terms of survival, and that is in contrast to a number of other reports. How do you explain the difference in your results compared with previous reports using that Royal College definition?

Dr Harvin. Well, our data are unique in that all patients in this analysis underwent neoadjuvant chemoradiotherapy. The other studies that have looked at this subject have either contained a very small percentage of patients receiving neoadjuvant chemoradiotherapy or none at all. We are in agreement with some other studies. For example, Deeter, published a report in 2009 that showed that the definition of a positive radial margin by the College of American Pathology definition was a more clinically-relevant definition. We certainly are in agreement with that study. Compared with some of the other studies that our results contradict, they only used the Royal College of Pathology definition. They did not differentiate their patients by tumor at the margin versus tumor at or within. Because we don't know the incidence or significance of tumor at the margin in those studies, we could not compare our results to theirs.

Dr Darling. Do you think that the radiation plays a significant role in your ability to achieve an R0 resection?

Dr Harvin. There is level 1 data that R0 resections are more common after neoadjuvant chemoradiotherapy rather than, say, neoadjuvant chemotherapy or surgery alone. We believe that the neoadjuvant chemoradiotherapy allows us to have more complete resections. This was, however, not a randomized study, so we cannot determine from this study what role radiation may play.

Dr Darling. My other question relates to your surgical approach. The majority of your patients had transthoracic resections. Do you think that had an effect on your results?

Dr Harvin. In terms of the data, we did not see any difference between the types of surgery and involved radial margins, so we cannot say that the type of surgery affected our results. The numbers for some of the different types of operations were small, however, so it could have been underpowered.

Dr Darling. I'm sorry, I should have made my question more clear. Do you think that that might explain the difference between your results and some of the other studies? 
Dr Harvin. I don't think we can answer that question from the data we have collected.

Dr Darling. You excluded patients with positive proximal or distal resection margins, but it seems there's only a very small number of patients in that group. Do you think that if we exclude patients with positive proximal and distal margins that it's very likely that the radial margin will also be negative? Can we use that as a predictor?

Dr Harvin. Although it's possible, the number of patients with positive proximal or distal margins and a positive radial margin were very few. So, I do not think we can use proximal or distal margin status as a marker for radial margin status.

Thank you, Dr. Darling, for your questions and for evaluating the manuscript.

Dr Brendon M. Stiles (New York, NY). Thank you very much. I enjoyed the talk.

I have two questions I was hoping to ask. One, can you predict the type of patients who are going to have positive margins? Some of that is by the type of surgery, but some also by the location of the tumor, by the histology of the tumor.

Dr Harvin. Can you repeat your question?

Dr Stiles. Are you able to get a sense of which patients are going to have positive margins going in? Is there a predictor; the type of surgery, whether they responded to induction therapy, the location of the tumor, gastroesophageal junction versus lower esophagus?

Dr Harvin. We do not have that analysis for the exact patients we presented today. However, an older multivariate analysis of similar ypT3 patients found that radiation doses greater than 45 Gy and pathologic lymph node status were independent predictors of a positive CRM (tumor at or within $1 \mathrm{~mm}$ of the radial margin).

Dr Stiles. My second question is how the patients responded to induction therapy. I know that in a lot of the publications from your institution, the response to neoadjuvant therapy has been a very powerful predictor of outcome. We have seen similar results. When we have tried to look at the effect of extended lymphadenectomy in this kind of group of patients, what we have seen is that in the responders it probably doesn't matter as much because they have a great systemic response and they are probably not going to have as bad an outcome. Conversely, in the nonresponders, we see that actually more aggressive local resection becomes more important. Have you looked at the clinical response and are you able to tell the difference between those two groups of patients?

Dr Hofstetter. In a multivariate analysis, both histoviability and response were included, and it wasn't an independent predictor. So we couldn't predict who was going to have a positive margin based on whether they had a response or not, and that's why, when we did the original analysis, we included all patients and all comers rather than focusing on just the ypT3s. The interesting finding in our data was that when we looked at the whole group, the CRM-positive patients actually performed slightly better than the CRM-negative ones. If you recognize the first Kaplan-Meier curve, we were above it-and that was including all patients who were ypTONOM0 as well.

In terms of your question about location or histology, those were both included and were not independent predictors, so we couldn't exactly delineate which were or which would not be. Now, I don't want to be the naysayer about the minimally invasive esophagectomy (MIE) procedure, but I will say that perhaps early in our learning curve, $50 \%$ of the patients who had undergone an MIE procedure had a close margin, although, according to the CAP definition, only 1 of 12 was positive. So this might, again, be early in our learning curve. It was not statistically significant, but if there was anything to delineate transthoracic versus transhiatal MIEs, which at that point we were doing a lot of, we had a few more close margins in that situation.

Dr Thomas W. Rice (Cleveland, Ohio). I have a comment about your analysis. If you continue to distill your data, eventually you're going to select out the worst people, and if you perform multiple, multiple tests, eventually one is going to be positive by chance. I have some advice. This was a retrospective study. You must match patients who don't have a positive resection margin with those who don't have a positive resection margin, because generally the people with the positive resection margins have the bigger tumors. And you can't compare a T1 to a T3. You have to compare a T3 and a T3, a T3 with a positive resection margin versus a T3 without. So you must propensity-match it and then you can tell us. You'll maximize the use of your data and you'll give us some very valuable information. Thank you very much.

Dr. Harvin. Actually, we did do a propensity score match and it showed no difference. We did not include that because our patients were so alike in the ypT3 group that we thought it wouldn't add much to the analysis.

Dr Rice. It's much better to include it. It will make it a much more valuable paper for the reviewers and the editors to see it.

Dr Harvin. Yes. Thank you, sir.

Dr Avi Lebenthal (Boston, Mass). I enjoyed your presentation.

The problem I have with this topic is the pathology; it's not really the surgery. What we have learned from the analysis of nodes in esophageal cancer is that when the Japanese cut them between 5 and 10 times, they get a very different incidence of node positivity than we do when we cut them once or when in Belgium they cut them 3 times. So it's very different in different places. It could be that the difference between the $1 \mathrm{~mm}$ and the positive margin is a function of also how the pathologist is looking at it. So if you really want to do this prospectively, I think, as a discipline, we have to sort of decide what the pathologist has to analyze in the tissue, because otherwise we're all collecting different data.

Dr. Harvin. Yes, sir.

Dr Mark J. Krasna (Towson, Md). I enjoyed your presentation, Dr. Harvin.

For you and Dr Hofstetter, there are data in rectal cancer that are very similar to this that I'm sure you're aware of if you looked at circumferential radial margins because it's the only other place we look at it routinely. The reason I bring it up is, with the new staging system, Wayne, you're always talking about what's the clinical application, how are we going to use it in clinical hands. What they do now in rectal cancer, based on the British data from multidisciplinary prospective reviews of MRIs, they predefine a subgroup of patients who might be likely to have a circumferential radial margin versus those negative, and this answers what Brendon was getting at. Do either of you have any thoughts about any of our current diagnostic testing, whether it's EUS or adding MRI in the esophagus, like rectal, that you might be able to predetermine that group of patients? 
Dr Hofstetter. That's a good question, Mark, and thank you for it. Our opinion in general, ongoing, is that we should be looking at a positive circumferential radial margin as the most significant one and that we need to be including that definition in our randomized trials. Whether or not it's at $1 \mathrm{~mm}$ or $2 \mathrm{~mm}$, which is what the colorectal surgeons have established, versus at the margin, I think might be site specific and it might also be treatment-specific. So we're trying to establish that CAP is the most relevant criteria and we're going to advocate using that going forward. In terms of what studies would be best for that, I don't know. I think that the pathologist makes a big difference, but also the surgery does too. I know that most of you, after you have done your transthoracic or modified en bloc esophagectomy don't go back and sew the pleura down to where it was over the tumor, you know, to say, well, don't disrupt this when it goes on its way over to pathology, and that might also have a significant effect on these close margins. The positive margins are positive likely because the surgeon can't go any further, meaning this is a T4a or it's into the pleura, and everything has been resected, and this is more of a marker of disease than a marker of surgery.

Dr Krasna. Can EUS or MRI-?

Dr Hofstetter. I don't know the answer to that. At our institution we had the guys doing EUS calling it T4a very frequently, and we did not use that as an indicator to change our therapy or change our operation, because not infrequently the call of T4 was incorrect or it wasn't borne out from the standpoint of surgery. Now, whether that's due to downstaging or not, I don't have the data for that.

Dr Scott Swanson (Boston, Mass). I really enjoyed that paper. In follow-up to what you just said, what do you do with those patients who have tumor at the margin? If you're giving them chemoradiotherapy, you know they are going to have a high chance of local recurrence as well as systemic recurrence. What's your treatment modality? Is there a radiation strategy, pure chemo strategy, IMRT? What do you do with them?

Dr Hofstetter. We entered into this project as a fact-finding mission. We didn't know quite what we were going to find with tumor close to the margin versus at the margin. As you saw, it was a fairly frequent finding for patients who were having a transmural viable tumor but didn't know whether it was at all significant.

I think it's interesting to note that the patients who have disease at the margin didn't do as well, but their local recurrences weren't any higher, and I think it's a marker for bad disease. Unfortunately, as you know, at this point there are several indicators for patients who are going to do poorly, none of which we know completely how to treat, but excess lymph node involvement, lymphovascular invasion, and I think disease at the margin is one of those other poor indicators, but of systemic disease, not necessarily of locoregional failure. So maybe that's something that we need to look at into the future. 